\title{
TERAPIA COMUNITÁRIA INTEGRATIVA PARA IDOSOS EM PLATAFORMA VIRTUAL DURANTE A PANDEMIA ASSOCIADA A COVID-19
}

\author{
TERAPIA COMUNITARIA INTEGRATIVA PARA ADULTOS MAYORES EN LA \\ PLATAFORMA VIRTUAL DURANTE LA PANDEMIA POR COVID-19
}
INTEGRATIVE COMMUNITY THERAPY FOR THE ELDERLY ON THE VIRTUAL PLATFORM DURING THE PANDEMIC ASSOCIATED WITH COVID-19

\author{
Dora Mariela SALCEDO-BARRIENTOS ${ }^{1}$ \\ Maria Vitória SILVA PAIVA ${ }^{2}$ \\ André Luiz Pereira da SILVA ${ }^{3}$
}

RESUMO: O presente estudo teve como objetivo compartilhar a experiência vivenciada junto a pessoas da terceira idade em plataforma virtual. Sustenta-se na estratégia prática da Terapia Comunitária Integrativa (TCI) e na Teoria Práxica de Enfermagem em Saúde Coletiva (TIPESC). A coleta foi realizada por meio de instrumentos específicos; foram realizadas 14 rodas com uma participação de 90 (100\%) idosos e foi realizada uma amostragem por conveniência. Dentre os principais resultados destaca-se a roda como uma tecnologia leve que pretende dar voz a este grupo etário considerado grupo de risco e contribuir para implementação de novas políticas no Brasil, avançando para inserir o idoso na nova tecnologia mundial, combatendo os desafios existentes e visando prevenir os impactos sócio-político-econômico após a pandemia associado a Covid-19.

PALAVRAS-CHAVE: Boas práticas. Saúde coletiva. Terapia comunitária integrativa. Envelhecimento. Família.

RESUMEN: Este estudio tuvo como objetivo compartir la experiencia vivida con personas de la tercera edad en una plataforma virtual. Se basa en la estrategia práctica de la Terapia Comunitaria Integrativa (TCI) y la Teoría Práxica de la Enfermería en Salud Pública (TIPESC). La recolección se realizó con instrumentos específicos; Se realizaron 14 ruedas con una participación de 90 (100\%) ancianos y se realizó un muestreo por conveniencia. Entre los principales resultados, la rueda se destaca como una tecnología ligera que tiene como objetivo dar voz a este grupo de edad considerado como un grupo de riesgo y contribuir a la

${ }^{1}$ Universidade de São Paulo (USP), São Paulo - SP - Brasil. Docente do Programa de Pós-Graduação em Enfermagem na Atenção Primária em Saúde no Sistema Único de Saúde (MPAPS). Pós-Doutoranda do Instituto de Saúde Pública da Universidade do Porto (ISPUP). Doutorado em Enfermagem (USP). Filiada a Associação Brasileira de Terapia Comunitária Integrativa (ABRATECOM). ORCID: https://orcid.org/0000-0003-1562-0598. E-mail: dorabarrientos@usp.br

${ }^{2}$ Instituto Afinando Vidas, São Paulo - SP - Brasil. Professora no Curso de Formação em Terapia Comunitária e Terapeuta Comunitária. Mestre em Relações Étnico Raciais (CEFET/RJ). Filiada a Associação Brasileira de Terapia Comunitária Integrativa (ABRATECOM). ORCID: https://orcid.org/0000-0002-6371-8648. E-mail: vitapaiv1@hotmail.com

${ }^{3}$ Instituto Afinando Vidas, São Paulo - SP - Brasil. Terapeuta Comunitário. Graduação em Psicologia (UNICASTELO). Filiado a Associação Brasileira de Terapia Comunitária Integrativa (ABRATECOM). ORCID: https://orcid.org/0000-0003-2641-1281. E-mail: a_luizp@yahoo.com.br 
implementación de nuevas politicas en Brasil, avanzando para insertar a los ancianos en la nueva tecnología mundial, combatiendo los desafios existentes. dirigido a prevenir impactos socio-político-económicos después de la pandemia Covid-19.

PALABRAS CLAVE: Buenas practicas. Salud pública. Terapia comunitaria integradora. Envejecimiento. Familia.

ABSTRACT: This study aimed to share the experience lived among elderly people on a virtual platform. It is based on the practical strategy of Integrative Community Therapy (ICT) and the Theory of Praxis Intervention of Nursing in Collective Health (TIPESC). The collection was carried out by means of specific instruments; 14 ICT circles with a participation of 90 (100\%) elderly people were carried out and a sample was taken for convenience. Among the main results, the circle stands out as a soft technology that intends to give voice to this age group considered a risk group and contribute to the implementation of new policies in Brazil, advancing to insert the elderly in the new world technology, fighting the existing challenges and aiming at preventing the socio-economic impacts after the pandemic associated to Covid19.

KEYWORDS: Good practices. Collective health. Integrative community therapy. Aging. Family.

\section{Introdução}

O processo de envelhecimento é um desafio mundial, segundo a Organização Panamericana da Saúde (OPS, 2019), o número global de pessoas idosas - entendidas com 60 ou mais anos de idade - está projetado para aumentar de 962 milhões em 2017 para 1,4 bilhão em 2030, todas as regiões do mundo, exceto a África, terão quase um quarto ou mais de pessoas idosas. Simultaneamente, segundo o Instituto Brasileiro de Geografia e Estatística (IBGE, 2018a) o Brasil tem mais de 28 milhões de pessoas nessa faixa etária, o que representa $13 \%$ da população do país, com projeção para dobrar nas próximas décadas.

O envelhecimento também é definido como um processo natural, progressivo e irreversível, próprio de seres viventes e que sem dúvidas recebe influência de fatores sociais, políticos, econômicos e psicológicos. Desta forma, as alterações decorrentes do envelhecimento influenciam pela sua vez os processos de comunicação, trazendo repercussões e consequente exclusão social (MESQUITA; CAVALCANTE; SIQUEIRA, 2016).

Segundo a Pesquisa Nacional de Saúde de 2013, mais recente do IBGE, 17,3\% dos idosos apresentavam limitações funcionais para realizar as Atividades Instrumentais de Vida Diária, aumentando para 39,2\% entre os de 75 anos ou mais (IBGE, 2013). Neste sentido, diante a pandemia causada pelo SARS Covid 19, a população teve que se reorganizar ante a imposição 
governamental de isolamento social, pensar no idoso dentro deste cenário inclusive a preocupação com a deficiente interação social se faz necessário, corroborando com o que orienta caderno de atenção ao Idoso da Prefeitura de São Paulo (SÃO PAULO, 2016).

As Práticas Integrativas Complementares (PICs) articulam e reforçam os princípios e diretrizes do Sistema Único de Saúde (SUS), visto que sua implementação respalda-se na promoção em saúde baseada na integralidade da assistência, com o enfoque na assistência humanizada. Sob uma perspectiva intersetorial e interdisciplinar, as práticas integrativas complementares, atuam no campo de prevenção de agravos e doenças, promovendo um cuidado holístico e progressivo, alinhado na singularidade de cada indivíduo. A implantação de estratégias como essa, é imprescindível na composição do envelhecimento saudável (BRASIL, 2006).

Para Lima (2007) é necessário que o idoso seja estimulado ao aprendizado de novas tecnologias. A internet é uma possibilidade de tirar o idoso de sua zona de conforto e colocá-lo em um caminho de novas aprendizagens que possam melhorar sua qualidade de vida.

A necessidade de comunicação nesta fase da pandemia demanda novas formas de pensar, além de entender esta fase do ciclo vital onde acontecem alterações de ordem fisiológica, há a necessidade de interpretar as modalidades que permeiam o processo interativo do idoso. Em paralelo ao envelhecimento populacional, tem-se a constante evolução das Tecnologias da Informação e Comunicação (TICs), as quais vêm se consolidando e mudando o perfil de comunicação entre os indivíduos.

Nesse sentido para que o idoso continue acompanhando esta evolução e não sofra impactos prejudiciais é adequado realizar a inserção na nova tecnologia de linguagem e comunicação monitorizando estas interações.

Diante esta nova conjuntura associada à Covid-19 muitos profissionais que atuam na atenção ao idoso enfrentaram este novo desafio, havendo a necessidade de reorganização e construção individual e coletiva de um novo processo de comunicação. Novas estratégias de interação foram sendo criadas para responder as necessidades de lidar com o sofrimento distanciamento social, culminando repensar as repercussões sócio-políticas, econômicas e prioritariamente de saúde mental.

Abordar o cuidado integral à população durante o envelhecimento é um desafio constante da sociedade brasileira e o momento é relevante para despender esforços para aplicação efetiva dos direitos humanos.

No Brasil, são diversas as desigualdades, dentre elas as condições de acesso a, trabalho, saúde, moradia, lazer, educação, desenvolvimento, que variam entre outros fatores, de acordo 
com a distribuição de renda, faixa etária, acesso à tecnologia e educação; disparidades essas, que acabam dificultando reinvindicações de alguns direitos, acesso a qualidade de vida, respeito à dignidade humana e envelhecimento digno.

\section{TCI como possibilidade da atenção integral e interação social}

Na Assembleia Geral da ONU, em 2015, foi estabelecido uma agenda para 2030 voltado ao desenvolvimento sustentável, que propõe 17 objetivos para transformar o nosso mundo, dialogando com o seguinte objetivo: Assegurar uma vida saudável e promover o bem estar para todas e todos, em todas as idades e contextos socioculturais. É enfatizado a responsabilidade de todos os Estados, em conformidade com a Carta das Nações Unidas, de respeitar, proteger e promover os direitos humanos e as liberdades fundamentais para todos, sem qualquer distinção de raça, cor, sexo, idioma, religião, origem nacional ou social, idade, deficiência ou outro determinante (ONU, 2015).

Neste contexto, a TCI constitui um espaço comunitário aberto a todos, amparada na promoção de saúde e centrada no alívio do sofrimento psíquico, na qual as pessoas prioritariamente idosas se encontram para compartilhar experiências de vida, pensar nas suas inquietações e, sobretudo, para construção de vínculos solidários. Considera-se a TCI um instrumento terapêutico que age acolhendo a dor através da escuta sem julgamentos ou apontamentos, dando visibilidade às dores e as formas de superá-las. Dessa forma, a implementação de ferramentas e políticas adequadas ao idoso devem ser estabelecidas, sendo elas respaldadas nos próprios objetivos da ONU.

Portanto, o presente estudo pretende compartilhar a experiência vivenciada junto a pessoas da terceira idade realizado durante a pandemia associada à covid-19 utilizando Rodas de TCI em plataforma virtual a partir de uma iniciativa de um polo formador na cidade de São Paulo.

\section{Metodologia}

Trata-se de um relato de experiência de 14 rodas junto aos idosos, realizadas durante o período de 27 de março a 26 de junho de 2020 por meio de uma plataforma digital, organizada pelo Polo formador em TCI Instituto Afinando a Vida (IAV) na cidade de São Paulo, reconhecido pela ABRATECOM. Sustenta-se na estratégia prática da TCI (BARRETO, 2015) e na Teoria Práxica de Enfermagem em Saúde Coletiva (TIPESC) (EGRY, 1996) que visa a 
sistematização dinâmica da captação e interpretação da realidade objetiva, articulado aos processos de produção e reprodução social para compreender o processo saúde-doença de um determinado grupo homogêneo em um contexto social e historicamente determinado.

Participaram destas rodas um total de 90 (100\%) idosos e fizeram parte desta amostra por conveniência especificamente 16 (18\%) participantes, os quais se caracterizaram por participar destas rodas de forma mais regular; dentre os instrumentos utilizados destaca-se uma lista de presença que continha dados sociodemográficos e uma ficha de avaliação preenchida após a execução de cada roda sobre a responsabilidade dos terapeutas comunitários, esta ficha continham dados relacionados com o número de participantes, temas prevalentes durante a roda; identificação com os temas abordados, estratégias de superação e avaliação do processo e sugestões para aperfeiçoar as seguintes rodas.

\section{Análise e Discussão dos Resultados}

\section{Perfil de produção e reprodução social}

Durante a execução das 14 rodas da TCI contou-se com a participação de 90 (100\%) idosos cuja idade encontrava-se no intervalo entre 60 e 83 anos, com predominância feminina, representada por 84 mulheres, 93\% dos participantes. e apenas $6(7 \%)$ homens.

Os dados a seguir foram obtidos a partir das informações dos 16 idosos que representam $18 \%$ do total dos participantes assíduos e para efeito desta apresentação serão contemplados $100 \%$ desta amostra por conveniência.

Quanto as formas de viver, a procedência da maior parte das idosas eram oriundas de várias partes do Brasil, com predomínio da cidade de São Paulo com representatividade das regiões norte, sul e leste, quanto a distribuição em território Nacional, observamos $81,25 \%$ dos idosos vivem na região Sudeste, $12,50 \%$ residem na região Nordeste e 6,25\% na região Norte.

Quanto a representação étnico racial, 50\% dos participantes se declararam brancos, $31,25 \%$ pardos e $18,75 \%$ pretos.

Quando indagados sobre os hábitos de vida e saúde, para ocupar o tempo livre do total de participantes, $68,75 \%$ se dedicam a culinária, $43,75 \%$ têm o hábito de leitura, $25 \%$ realizam caminhadas e 37,5\% desenvolveu alguma atividade manual. Nos aspectos de saúde há prevalência de doenças crônicas como Hipertensão Arterial e Diabetes Mellitus (50\% e 18,75\% respectivamente), 12,5\% faz acompanhamento para alguma neoplasia e 6,25\% tratam dislipidemia e depressão. 
Em relação ao grau de instrução 56,25\% ensino superior, $12,5 \%$ cursaram parcialmente o ensino superior, $18,75 \%$ concluíram o ensino médio, 6,25\% não foram alfabetizados.

Quanto a moradia, 62,25\% dos participantes residiam em domicílio com até três pessoas, $25 \%$ moram sozinhos, 6,25\% convivem com até cinco pessoas, sendo esta representatividade equivalente à dos idosos que moram com mais de cinco pessoas. Quanto as condições de habitabilidade, 87,5\% residem em casas próprias de alvenaria e os demais em habitações coletivas. Horta e Daspett (2018) apontam que o número de idosos que moram sozinhos vem crescendo no Brasil, fato este atrelado ao envelhecimento da população, pesquisa divulgada pelo IBGE (2018b), aponta que no Brasil 25\% dos idosos residem com três ou mais moradores, $60 \%$ com até duas pessoas e outros $15 \%$ estão sozinhos em suas casas.

Todos os participantes afirmam que a religião é um aspecto importante, inclusive os que não declararam seguir qualquer tipo de instituição religiosa representado por $12,5 \%$ da amostra, os católicos representavam $62,25 \%$ do grupo, seguidos pelos espíritas $18,75 \%$ e em minoria os budistas $6,25 \%$.

Quanto as formas de trabalho, os aposentados representam 43,75\% do grupo, sem a realização de outras atividades laborais, $25 \%$ apesar da aposentadoria exerciam outras atividades profissionais ou domésticas. Considerando-se o sustento financeiro, $25 \%$ dos participantes recebem até 2 salários mínimos, 62,5\% recebem o equivalente de 2 a 5 salários mínimos, e apenas $12 \%$ tem renda superior a 5 salários mínimos.

A partir da ótica da determinação social, com a interpretação do processo saúde doença atrelado a questões sociais relacionadas a hábitos individuais, formas de ocupação, bem como contexto social, percebe-se que a questão do envelhecimento constitui fator deletério como determinante singular, devido alterações dinâmicas e contínuas, que impactam na maneira de interagir socialmente. Em contrapartida, a participação nas rodas de TCI, de forma voluntária promove um fator protetor no sentido em que estes participam de atividades em grupo, com maior responsabilidade no cuidado de si, a partir do reconhecimento de suas limitações estimulado pelo compartilhamento das experiências neste espaço de comunicação coletiva.

Em relação ao grau de escolaridade apresentado, constatou-se que as taxas de escolaridade aqui citadas, diferem do perfil sociodemográfico dos idosos brasileiros. Segundo a Pesquisa Nacional por Amostra de Domicílios Contínua (PNAD Contínua) de 2019, a taxa de analfabetismo dos indivíduos com 60 anos ou mais era de 18,0\%. Nota-se ainda que, no Brasil, a taxa de analfabetismo está diretamente relacionada à idade. Logo, quanto mais velho o grupo populacional, maior é a proporção de analfabetos (IBGE, 2019).

O percentual de pessoas de 25 anos ou mais, com o ensino superior completo, passou 
de 16,5\% em 2018 para 17,4\% em 2019, no território brasileiro. Dessa forma, evidencia-se o alto nível de escolaridade do público alvo deste estudo, superando até mesmo o nível educacional de adultos e jovens, em âmbito nacional (IBGE, 2019).

A alta escolaridade apresentada pelos idosos do grupo demonstra ser um fator protetor, dentro das dimensões singular e estrutural, uma vez que muitos estudos trazem o fator escolaridade alta como fator de proteção mediante a inúmeros desfechos. Um estudo realizado para apurar os fatores individuais e contextuais associados à incapacidade em idosos brasileiros apontou que a baixa escolaridade e a ausência de rede social/familiar tiveram associações a incapacidades. Além de pontuar também que problemas para acessar os serviços de saúde podem ampliar as chances de incapacidade (FIGUERÊDO, 2019).

No que diz respeito às formas de viver, foram trazidas atividades como cozinhar, ler $\mathrm{e}$ caminhar para a ocupação do tempo livre, práticas essas que se evidenciam como processos protetores. Para Rocha e Grabosque (2019), as atividades de lazer dos idosos e as suas autopercepções de saúde, contribuíram para preservação da autonomia e independência e o seu bem estar físico e cognição.

Relativo às formas de trabalho a maioria dos idosos encontram-se aposentados $\mathrm{e}$ 43,75\%, parte significativa, além de aposentado exerce algum tipo de atividade laboral. A aposentadoria simboliza a transição entre o universo laboral e outra fase da vida, em que há um rearranjo dos papéis sociais. Ademais, ela representa um elemento fundamental do Estado de Bem Estar Social, promovendo maior qualidade de vida a população idosa junto a seguridade social. Dessa forma, pode-se inferir que a aposentadoria atua como um fator protetor dentro da dimensão singular, uma vez que se associa e impacta na saúde do indivíduo. Na dimensão estrutural, também tem função protetiva ao que consiste em uma política essencial para a saúde e qualidade de vida dos idosos.

\section{Experiências vivenciadas durante as Rodas de Terapia Comunitária em ambienta virtual}

De forma a possibilitar a continuidade das rodas de TCI durante a pandemia causada pelo SARS-covid-19, que culminou na imposição de distanciamento social, houve a necessidade de adaptar os encontros para o ambiente virtual, tendo em vista suprir as necessidades dos participantes promovendo um ambiente de acolhimento, apoio e integração.

O pioneirismo dessa ação se fez pelo IAV em parceria com o Núcleo do Centro de Convivência do Idoso (NCI) São Francisco de Assis na cidade de São Paulo. O NCI, serviço de proteção social, convivência e fortalecimento de vínculos aos idosos com idade igual ou 
superior a 60 anos em situação de vulnerabilidade e risco pessoal e social. Oferece atividades socioeducativas planejadas baseadas nas necessidades, interesses e motivações dos idosos, conduzindo na construção e reconstrução de suas histórias e vivências individuais e coletivas, na família e no território o mesmo que promove a vinculação com idosos a partir de acompanhamentos individuais e grupais, este serviço é composto por equipe multidisciplinar e multiprofissional.

\section{É chegada a hora de falar}

Canta canta, minha gente Deixa a tristeza prá lá Canta forte, canta alto Que a vida vai melhorar (Martinho da Vila)

A canção acima dialoga com a frase de Barreto (2008) que diz que "quando a boca cala os órgãos falam e quando a boca fala os órgãos sara". A fala é uma das formas de expressão na TCI, mas não a única, os participantes podem se expressar através de música, poesia, por provérbios, ditos populares e piadas. Nesse sentido, um dos pilares da TCI é a antropologia cultural ou as nossas raízes culturais.

\section{Temas abordados nos encontros semanais}

De acordo com Barreto (2015) a comunidade tem o problema, ela também tem a solução. O que ficou evidente durante a realização dos encontros virtuais.

É de suma importância a valorização e respeito a todo participante de uma roda de TCI, se o contrário fosse verdade, estaríamos a excluir e não acolhendo o indivíduo. Traríamos para roda um determinante social como forma de validar nossa autoexclusão. Sendo assim, a participação de cada um é muito relevante, pois a partir de sua fala ocorre a transformação intrínseca do indivíduo.

Cada participante é corresponsável pela qualidade afetiva das rodas, pois, o ato de transformar-se faz com que a pessoa desenvolva a habilidade e escuta, a partir do ato de permanecer em silêncio. Sendo assim, as pessoas podem falar e escutar umas às outras, exercitando uma forma diferente de hierarquia, a horizontalidade e com foco no enfoque sistêmico e a circularidade.

A seguir, destacamos alguns dos temas abordados nos encontros. 


\section{Saudades}

Uma das participantes relatou a falta que sentia dos netos, sendo este o tema escolhido pelo grupo. No momento de partilha das superações, um senhor contou a sua estratégia para matar a saudade que estava sentindo do neto e da mãe que está com mais de 90 anos de idade; ele relatou que compraria uma capa de chuva, luva e touca para se encontrar com o neto e com a mãe. Todo cuidado para não contaminar a mãe e prevenir o risco de ser contaminado pelo neto.

\section{Pandemia}

Dos temas abordados nos encontros, $71,4 \%$ estão diretamente relacionados a pandemia em decorrência do covid-19. As preocupações, incertezas e inseguranças, relatadas pelos idosos e referentes ao medo de familiares que trabalham na área da saúde, serem infectados, desconhecimento do vírus, com o futuro após a pandemia, saudade de familiares, perda de entes queridos não vítimas de covid-19; saudades dos netos. Barreto et al. (2020) abordaram a Terapia Comunitária Integrativa em tempos do novo Coronavírus em um recente artigo, na qual os temas levantados foram similares com os aqui apresentados. Dentre as 100 rodas online realizadas, os 3 tópicos mais frequentes foram: medo e ansiedade; impotência; e dificuldade em administrar relações familiares nesse novo contexto mundial.

Segundo a Cartilha Saúde Mental e Atenção Psicossocial na pandemia COVID-19, lançada pela Fiocruz, os brasileiros podem sofrer impactos psicológicos e sociais em vários níveis de intensidade e gravidade advindo de todo esse contexto em que o mundo se encontra (FIOCRUZ, 2020). O medo, o estresse, a ansiedade, junto aos anseios e a inviabilização de interações sociais são grandes fatores que marcam o período pelo qual nos encontramos. Esses entre outros sentimentos podem ser ainda mais intensificados na condição de isolamento social.

Outra situação a ser superada é a mudança da rotina familiar que gera necessidade de flexibilidade e adaptação, falou-se também sobre a dificuldade de lidar com a limitação na sua autonomia e estar aprendendo a receber a ajuda de familiares que fazem compras e as demais atividades externas. A preocupação em dar trabalho para alguém é uma condição que assombra os idosos, circunstância essa que reflete o medo da ausência de autonomia, e a ressignificação da pessoa idosa na sociedade (PERSEGUINO; HORTA, 2018). 


\section{Outros temas}

Os demais temas estão relacionados a conflitos no relacionamento familiar, perda por morte não decorrente do Corona vírus e a tristeza por não poder participar das festividades juninas.

\section{Ressignificação de problemas}

A seguir serão apresentadas algumas estratégias que contribuíram na resolutividade ou na redução do estresse, do medo e das angústias apresentadas pelos participantes e dentre elas destacam-se o uso tecnologias de informática e da internet.

Os relatos vão desde a participação em lives de diversos temas, grupos de dança, yoga, meditação, realização de trabalhos voluntários, até ao acesso às redes sociais para manterem a esperança viva, interagirem com amigos e familiares para vencer a saudade e o distanciamento. Houve a criação de grupos no whatsapp e no facebook para interagir.

A participação em grupos de dança que promoveram o contato com novos talentos em tempos de crise.

Apesar do distanciamento social a internet promoveu a ajuda religiosa e espiritual, tratamentos psiquiátricos e psicológicos, participação nos encontros de rodas de terapia comunitária.

Neste sentido, evidencia-se o distanciamento e isolamento social como um processo ambíguo (protetor e destrutivo). As medidas adotadas representam primeiramente, um fator protetor, essencialmente, para a população idosa, que se encontra no grupo de risco, uma vez que é uma recomendação estratégica e imprescindível para controle de contágio. Por outro lado, temos essas medidas de proteção como um processo destrutivo, tendo elas como potencial agente causador de problemas psicossociais, que afetam direta e principalmente a terceira idade, tendo em vista que essa população, geralmente, demanda maior cuidado, seja suporte afetivo/emocional, ou físico, dado suas limitações.

\section{Encerramento: Avaliação do Processo: O que estou levando desta roda?}

É o momento de ressaltar os esforços individuais na busca de lidar com os desafios cotidianos que, em muitos casos já estão ocorrendo, mas que a angústia é um obstáculo para 
enxergar, também é importante reconhece e a habilidade do grupo em acolher o outro com o seu sofrimento.

As pessoas que participaram das rodas de terapia comunitária saíram diferentes e apesar da fragilidade de sentimentos, mostraram-se mais confiantes, fortes e muitos deles mais certos do que foram e as possibilidades de mudar para melhor em suas vidas, mais seguro de si e do que acreditam.

Durante o ritual de agregação, considerada a última etapa da TCI, os participantes são estimulados a verbalizar o que estão a levar desta experiência. Palavras que foram citadas: gratidão (12), coragem (6); esperança e solidariedade (5), alegria, fortaleza, acolhimento, fé e partilha (3), sabedoria, amizade, paciência, compaixão (2); reflexão, valorização, dedicação, acalento, força, fidelidade, compartilhamento, aconchego, amor, conhecimento, resiliência, superação, confiança, encorajamento, companheirismo, bons momentos (3), pensar positivo (2), escutada e força, valorização do tempo, visitar as memórias, saber que não está sozinha, atenção e solicitude, um pouco de cada um, riqueza do grupo, valorização do tempo, ser útil, juntos somos fortes, somos um só, doar e receber, mais experiência, a paciência com o pincel para pintar, pensar positivamente, prazer em receber, ser amada, lições de fidelidade, solicitude do grupo, estar juntas em tempo de pandemia, gratidão pelos testemunhos, apoio do grupo, empoderamento pessoal, novo aprendizado, mais experiência.

Nesta perspectiva, o processo saúde doença é o resultado do contraste entre os processos protetores e os processos destrutivos no que tange as dimensões singular, particular e estrutural. A análise sociodemográfica permite evidenciar quais os processos protetores e destrutivos do público deste estudo e, complementarmente, a análise dos achados durante as rodas permite evidenciar potentes processos protetores que fortalecem este processo saúde como um direito adquirido e que se fortalece coletivamente.

\section{Fortalecimento das redes sociais: Criação de um grupo no whatsapp}

Neste tempo de planejamento, execução e organização das rodas foi importante que a equipe sempre esteve alerta a novas estratégias que contribuíram na resolutividade ou na redução do estresse, do medo e das angústias apresentadas pelos participantes dentre as quais destaca-se a criação de um grupo no whatsapp especificamente com esta finalidade de manter aquecido o grupo para dar sequência a um novo encontro, cujo impacto foi positivo com participação ativa, por talvez ser uma ferramenta amigável, em um ambiente de respeito e plena 
interação mais acessível à terceira idade. Acredita-se nesta potencialidade desta ferramenta que poderia ser melhor explorada.

\section{Novos desafios: Ser terapeuta comunitário em tempo de pandemia junto aos idosos}

Os dados apresentados fortalecem as metas a serem alcançadas na atenção saúde da pessoa idosa conforme o proposto pela Política Nacional de Saúde da Pessoa Idosa (PNSPI), que traz a capacidade funcional como um novo paradigma de saúde, com foco na independência e a autonomia, pelo maior tempo possível.

Os diversos sentimentos e emoções vivenciados por esta população sugerem $\mathrm{o}$ investimento em um trabalho muito mais pontual e articulado com a Atenção Primaria de Saúde (APS) no primeiro nível de atendimento e os NCI para trabalhar estas demandas. Com isso, nossa pergunta é como as instituições formadoras estão trabalhando estas questões durante a formação? Como está sendo trabalhado estes conteúdos nas diversas grades curriculares onde a população alvo é justamente o idoso? E como vem sendo abordados, inclusive durante a formação do futuro terapeuta comunitário, estes conteúdos? E sobretudo qual é o sentimento do futuro terapeuta em relação a este público alvo no seu cotidiano e, fundamentalmente, hoje em tempo de pandemia? O que ficou visível hoje?

Outro ponto importante de ressaltar é o impacto vivenciado pelos idosos em decorrência da restrições do governo foram relatadas nas rodas virtuais realizadas, o que reforça a necessidade de implementação de instrumentos mais eficazes que instrua o idoso na linguagem da nova tecnologia possibilitando a comunicação, melhor interação social e qualidade de vida.

Através das rodas de terapias online, realizadas durante o período de quarentena em São Paulo, ficou evidente a deficiência de projetos governamentais para a inserção do idoso na era tecnológica e desassistência para as novas tendências, sendo uma questão de enfrentamentos na atenção pública.

Esta experiência representa talvez uma das experiências mais profundas e significativas no cotidiano profissional vivenciado pelos terapeutas, pois permitiu uma aproximação quase natural justamente em tempo de pandemia, quando esta população alvo precisava ficar enclausurada em nome da sua segurança.

Representou uma oportunidade de ouvir nos relatos de experiência, alguns reflexos do cenário de envelhecimento diante de limitações, sentimentos profundos; dar voz a esta população nos desperta para repensar a nossa práxis, pois são necessárias também novas 
mudanças de rotina, as quais ficaram mais evidentes nesse período de quarentena pandemia mundial.

Portanto estabelecer uma articulação com outros NCI com esta proposta de atendimento com tecnologia leve e fundamentalmente implementar rodas de terapia comunitária de forma virtual, nos possibilitaria ofertar um atendimento amplo dando suporte de forma integral às necessidades de saúde física, mental e social.

\section{Conclusão}

O presente estudo representou um espaço de novas e profundas reflexões sobre a importância do curso de formação em TCI para abordar de forma mais aprofundada as fases do ciclo vital com ênfase nos idosos desde uma perspectiva da determinação social, pois como foi evidenciado é um grupo que está crescendo no Brasil e existe a necessidade de novos profissionais qualificados técnica e emocionalmente falando para trabalhar com essa parcela da população.

Assim também percebe-se a importância de implementação de mais recursos governamentais em apoio aos programas e políticas públicas conforme perfil de produção e reprodução social na população, com atividades que auxiliem e incentivem a inserção social do idoso na linguagem da comunicação das novas tecnologias, bem como atividades físicas e medidas preventivas de saúde de baixo custo ou gratuito, possibilitando que a população possa exercer sua cidadania e preservar a proteção dos seus direitos.

Dentro desta ótica, da determinação social, foi evidente identificar o grande número de pessoas idosas morando sozinhas, a nossa amostra demonstrou essa realidade, sugere-se pensar em novas estratégias para lidar com as medidas de isolamento físico.

Este isolamento físico que também se tornou social, por um lado representou um processo destrutivo e seria importante torná-lo protetor para facilitar esta aproximação familiar pois esse afastamento do convívio foi forçado pela conjuntura, no entanto existem limitações pessoais que impossibilitam o deslocamento dessas pessoas para realizar tarefas cotidianas. Sugere-se equipar os NCIs para essa tarefa, acompanhar de forma efetiva aos idosos nas suas necessidades básicas e, especialmente, aqueles que não tem condições de se deslocar até o equipamento e monitorar estas ações.

O desenvolvimento de atividade coletiva em plataforma virtual para o público idoso, neste caso a TCI evidenciou um processo destrutivo na dimensão singular e particular na sociedade brasileira, isto é a dificuldade das pessoas que tem baixo poder aquisitivo de se 
beneficiarem dessa oportunidade devido a pelo menos três fatores; um deles a falta de habilidade com a tecnologia na população acima de sessenta anos, a falta de um aparelho celular ou computador para acessar a internet nos próprios domicílios, e por último e não menos importante, a falta de condições de pagar por um plano de internet que possibilite o acesso às plataformas digitais, visto que o poder público não disponibiliza esse serviço.

A criação de um grupo no whatsapp foi mais uma estratégia de acolhimento para os novos participantes, constituindo-se em um espaço para troca de ideias, conversas informais e inclusive proporcionando uma maior facilidade de acesso a esta rede pelo público beneficiado, o que nos levou considerar a viabilidade de desenvolver a TCI neste ambiente virtual e melhorar a comunicação com o grupo, pois observamos que esse recurso pode ser otimizado.

Foi possível constatar também em muitos casos que a internet é precária, o que dificulta a participação com qualidade do idoso na roda, interrompendo a conexão e sugere-se também serem realizadas algumas parcerias com outras instituições públicas e privadas e aumentar a divulgação destas iniciativas saudáveis.

Finalmente, essa bela poesia musicada: "Sei lá (A vida tem sempre razão) de Tom Jobim" cumpre muito bem a função de dizer em versos a rica experiência que foi para nós, terapeutas comunitários, os encontros com os idosos. Esse contato nos aproximou ainda mais dos nossos ancestrais, esse resgate foi possível porque aprendemos juntos, a sabedoria dos mais velhos que nutriu a todos, participantes e terapeutas comunitários numa fluidez natural e permitiu que este momento seja de grandes aprendizados coletivos.

\section{REFERÊNCIAS}

BARRETO, A. P. Terapia comunitária: passo a passo. 4. ed. rev. ampl. Fortaleza, CE: Gráfica LCR, 2008.

BARRETO, A. P. Terapia comunitaria integrativa paso a paso. Quito, Ecuador: Digital Center, 2015.

BARRETO A. P. et al. Integrative community therapy in the time of the new coronavirus pandemic in Brazil and Latin America. World Soc. Psychiatry, v. 2, n. 2, p. 103-105, 2020.

BRASIL. Ministério da Saúde. Secretaria de Atenção à Saúde. Departamento de Atenção Básica. Política Nacional de Práticas Integrativas e Complementares no SUS - PNPICSUS. Brasília: Ministério da Saúde, 92 p., 2006.

EGRY, E. Y. Saúde coletiva: construindo um novo método para a enfermagem. São Paulo: Ícone, 1996. 
FIGUERÊDO, D. S. T. O. Fatores individuais e contextuais associados à incapacidade em idosos brasileiros. 2019. 146 f. Tese (Doutorado em Enfermagem) - Universidade Federal de Minas Gerais, Belo Horizonte, 2019.

FIOCRUZ. Fundação Oswaldo Cruz. Saúde Mental e Atenção Psicossocial. Recomendações Gerais. 8 p., 2020. Disponível em: https://www.fiocruzbrasilia.fiocruz.br/wpcontent/uploads/2020/04/Sa\%c3\%bade-Mental-e-Aten\%c3\%a7\%c3\%a3o-Psicossocial-naPandemia-Covid-19-recomenda\%c3\%a7\%c3\%b5es-gerais.pdf. Acesso em: 27 jul. 2020.

HORTA, A. L. M.; DASPETT, C. (Orgs.). Desafios no trabalho com famílias: da teoria à prática. Curitiba: CRV, 2018.

IBGE. Instituto Brasileiro de Estatística e Geografia. Pesquisa Nacional de Saúde (PNS). Rio de Janeiro, 2013. Disponível em: https://censo2020.ibge.gov.br/2012-agencia-denoticias/noticias/24036-idosos-indicam-caminhos-para-uma-melhor-idade.html. Acesso em 27 jul. 2020.

IBGE. Instituto Brasileiro de Estatística e Geografia. Projeção da população brasileira. Rio de Janeiro, 25 jul. 2018a.

IBGE. Instituto Brasileiro de Estatística e Geografia. Número de idosos cresce $18 \%$ em 5 anos e ultrapassa 30 milhões em 2017. Agência IBGE Notícias, Rio de Janeiro, 26 abr. 2018b. Disponível em: https://agenciadenoticias.ibge.gov.br/agencia-noticias/2012-agencia-denoticias/noticias/20980-numero-de-idosos-cresce-18-em-5-anos-e-ultrapassa-30-milhoes-em2017. Acesso em: 15 jul. 2020.

IBGE. Instituto Brasileiro de Estatística e Geografia. Pesquisa Nacional por Amostra de Domicílios Contínua (PNAD Contínua) de 2019: taxa de analfabetismo. Rio de Janeiro, 2019. Disponível em:

https://biblioteca.ibge.gov.br/visualizacao/livros/liv101736_informativo.pdf. Acesso em: 15 jul. 2020.

MESQUITA J. S.; CAVALCANTE, M. R. L.; SIQUEIRA, C. A. Promoção da saúde e integralidade na atenção ao idoso: uma realidade brasileira. Revista Kairós Gerontologia, v. 19, n. 1, p. 227-38, 2016.

ONU. Organização das Nações Unidas. Agenda 2030 para o Desenvolvimento Sustentável. 2015. Disponível em: https://nacoesunidas.org/pos2015/agenda2030/. Acesso em: 6 jul. 2020.

OPS. Organización Panamericana de la Salud. Plan of action on the health of older persons, including Active and healthy aging: final report. 2019. Disponível em: https://www.paho.org/hq/index.php?option=com_docman\&view=download\&alias=49691 cd57-inf-9-e-poa-older-persons\&category_slug=cd57-en\&Itemid=270\&lang=en. Acesso em: 10 jul. 2020.

ROCHA, A. R. G.; GRABOSQUE, C. L. Análise das condições de saúde autorreferidas e práticas de lazer em idosos assistidos na atenção primária. 2019. 18 f. Monografia (Trabalho de Conclusão do Curso de Enfermagem) - Centro Universitário de Maringá, Maringá, PR, 2019. 
SÃO PAULO. Secretaria Municipal da Sa de de São Paulo. Coordenação da Atenção Básica. Área Técnica de Sa de da Pessoa Idosa. Documento norteador unidade de refer ncia sa de do idoso. São Paulo, 2016.

\section{Como referenciar este artigo}

SAlCEDO-BARRIENTOS, D. M.; SIlVA PAIVA, M. V.; SILVA, A. L. P. da. Terapia Comunitária Integrativa para idosos em plataforma virtual durante a pandemia associada a Covid-19. Temas em Educ. e Saúde, Araraquara, v. 16, n. esp. 1, p. 360-375, set., 2020. eISSN 2526-3471. DOI: https://doi.org/10.26673/tes.v16iesp.1.14317

Submetido em: 20/05/2020

Revisões requeridas: $30 / 05 / 2020$

Aprovado em: 25/08/2020

Publicado em: 30/09/2020 